\title{
Large-area synthesis of a semiconducting silver monolayer via intercalation of epitaxial graphene
}

\author{
Philipp Rosenzweig* and Ulrich Starke ${ }^{*}$ \\ Max-Planck-Institut für Festkörperforschung, Heisenbergstraße 1, 70569 Stuttgart, Germany
}

(Received 16 March 2020; revised manuscript received 30 April 2020; accepted 4 May 2020; published 20 May 2020)

\begin{abstract}
Two-dimensional monolayers consisting of a single element have attracted considerable interest due to their intriguing properties, which can be fundamentally different from the bulk counterparts. However, their largescale synthesis often remains challenging owing to the nonlayered nature of the respective bulk crystal structures. In this Rapid Communication we show that the noble metal silver can be confined into the monolayer limit via intercalation between silicon carbide and epitaxial buffer layer graphene. Using angle-resolved photoelectron spectroscopy we reveal the formation of a silver-related valence band whose dispersion can be described by a simple, triangular-lattice tight-binding model. Interestingly, the synthesized silver monolayer is semiconducting as opposed to the prototype $s p$ bulk metal. The intercalation process further yields an $n$-type doped quasi-freestanding graphene monolayer, thereby realizing a two-dimensional metal/semiconductor heterostructure. Our results demonstrate the potential of epitaxial graphene on silicon carbide as a functional platform for the waferscale synthesis of monoelemental monolayers with unique attributes.
\end{abstract}

DOI: 10.1103/PhysRevB.101.201407

\section{INTRODUCTION}

Two-dimensional (2D) materials are a current focus of condensed matter physics $[1,2]$ due to their unique properties which are absent in the bulk homologs and make them promising candidates for, e.g., next-generation (opto)electronics and catalysis [3-5]. Unfortunately, the large-scale epitaxy of monoelemental monolayers (MLs) typically requires delicate procedures owing to the nonlayered bulk crystal structures [6]. Only few such materials beyond graphene have so far been realized [7-12]. Despite their application potential the noble metals have proven particularly intractable as they tend to form 2D nanostructures of limited lateral extent [13-15].

In Refs. [7-12] the appropriate choice of substrate was crucial for the successful ML growth. For graphene, silicon carbide ( $\mathrm{SiC}$ ) stands out as an epitaxy template since it facilitates large-area sublimation growth of high quality [16-18]. The properties of the as-grown graphene can further be tuned via intercalation of foreign atomic species at the graphene/ $\mathrm{SiC}$ interface-cf., e.g., Refs. [19-26].

While the majority of intercalation studies focus on graphene, the intercalants' electronic (and atomic) structure has largely been overlooked. Only recently tin $[27,28]$ and gold [29] were shown to form a triangular lattice ML when intercalated at the graphene/ $\mathrm{SiC}$ interface, yielding dispersive electronic bands. The $\mathrm{SiC}$ substrate defines the epitaxial order

\footnotetext{
*p.rosenzweig@fkf.mpg.de
}

Published by the American Physical Society under the terms of the Creative Commons Attribution 4.0 International license. Further distribution of this work must maintain attribution to the author(s) and the published article's title, journal citation, and DOI. Open access publication funded by the Max Planck Society. of the intercalated MLs, yet only graphene guarantees their stability via vertical confinement. The intercalant is further protected from the environment such that ex situ applications appear more likely. Epitaxial graphene on $\mathrm{SiC}$ might therefore be an ideal platform for the scalable synthesis of various monoelemental MLs with distinct electronic structures. To highlight this general potential the spectrum of available materials should be extended. The noble metal silver ( $\mathrm{Ag}$ ) appears promising, adopting a layered geometry upon plasma-assisted intercalation [30]. Yet, experimental evidence of concomitant band structure formation is still lacking.

In this Rapid Communication we intercalate $\mathrm{Ag}$ at the interface of $\mathrm{SiC}$ and its $(6 \sqrt{3} \times 6 \sqrt{3}) \mathrm{R} 30^{\circ}$ carbon buffer layer via conventional solid state evaporation. The encapsulated atoms form a triangular lattice and an associated valence band develops, peaking well below the Fermi level as probed by angle-resolved photoelectron spectroscopy (ARPES). We thus demonstrate an unambiguous metal-to-semiconductor transition when confining Ag from bulk into the ML limit. A 2D tight-binding model largely reproduces the dispersion while hybridization with $\mathrm{SiC}$ bands near $\bar{\Gamma}$ and core level spectroscopy indicate a finite electronic interaction with the substrate. The intercalation process further transforms the carbon buffer layer into $n$-doped quasi-free-standing graphene, creating a well-defined 2D metal/semiconductor heterostructure.

\section{EXPERIMENT}

Preparation of the $6 \mathrm{H}-\mathrm{SiC}(0001)$ substrate and growth of the $(6 \sqrt{3} \times 6 \sqrt{3}) \mathrm{R} 30^{\circ}$ carbon buffer layer [zerolayer graphene (ZLG)] are described elsewhere [18,25,31]. About $6 \mathrm{~nm}$ of silver is evaporated in situ from a commercial Knudsen cell onto ZLG at room temperature, followed by stepwise annealing up to $650^{\circ} \mathrm{C}$ for at least $30 \mathrm{~min}$ [32]. 

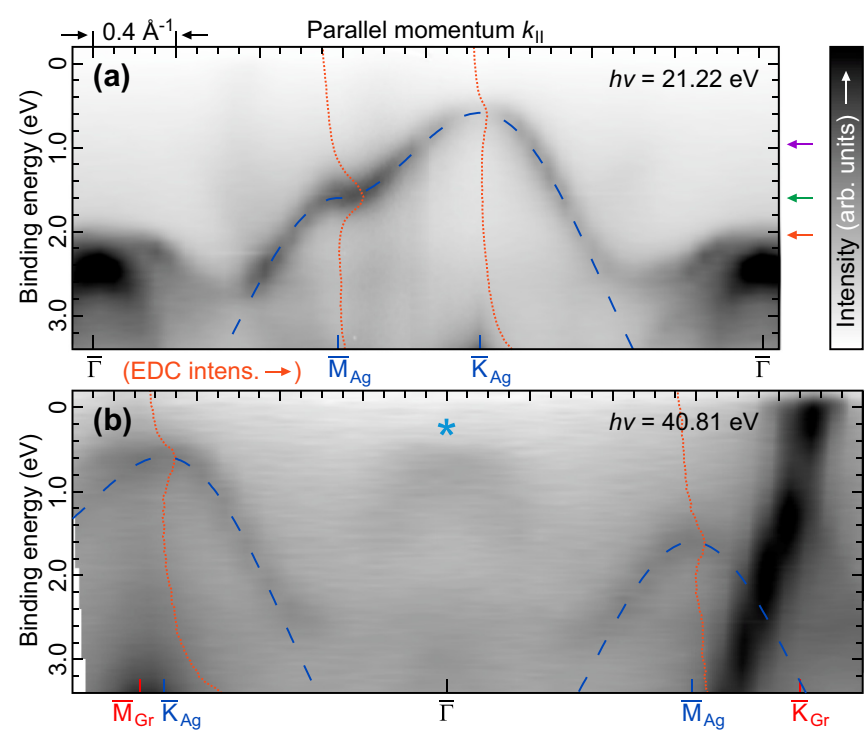

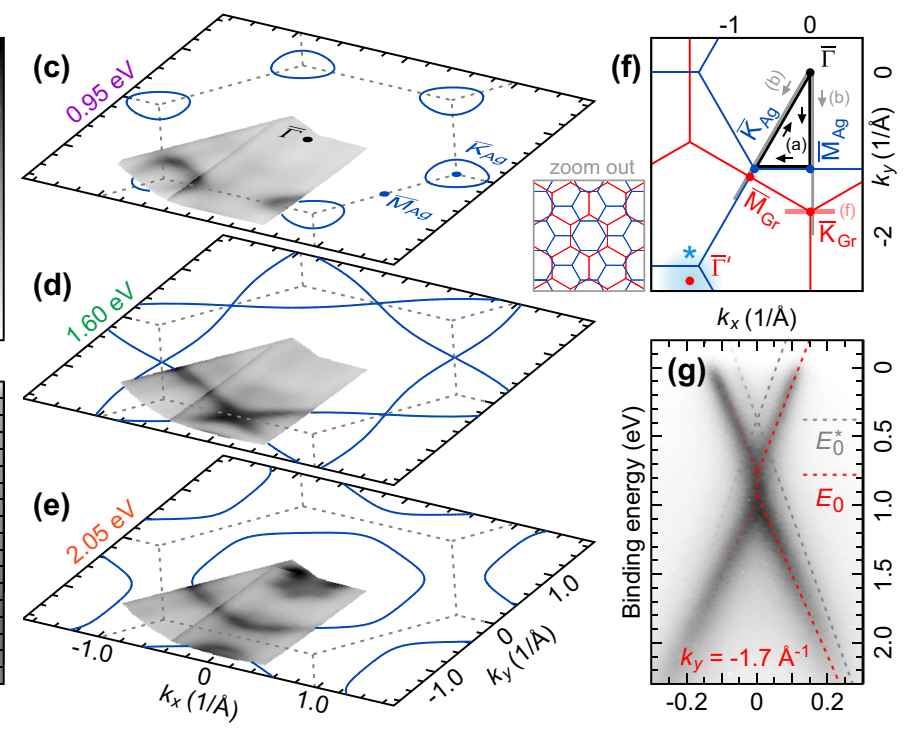

FIG. 1. Electronic structure of Ag-intercalated ZLG probed by ARPES. (a) Dispersion along $\overline{\Gamma M K \Gamma}$ in the first Ag BZ. The dashed blue curve represents a next-nearest-neighbor TB model fitted to the Ag-induced valence band away from $\bar{\Gamma}$. Energy distribution curves (EDCs) at $\overline{\mathrm{K}}_{\mathrm{Ag}}$ and $\overline{\mathrm{M}}_{\mathrm{Ag}}$ are overlayed (dashed orange). (b) Dispersion along $\overline{\mathrm{M}}_{\mathrm{Gr}} \mathrm{K}_{\mathrm{Ag}} \Gamma \mathrm{M}_{\mathrm{Ag}} \mathrm{K}_{\mathrm{Gr}}$ (logarithmic color scale). (c)-(e) Isoenergy cuts through the Ag valence band and respective TB contours at three different binding energies. (f) Alignment of the hexagonal BZs of Ag and graphene and orientation of the ARPES cuts (a), (b), and (g). (g) $\pi$ bands of QFMLG (dashed red, $E_{0} \approx 0.8 \mathrm{eV}$ ) perpendicular to $\overline{\Gamma K}_{\mathrm{Gr}}$. The faint Dirac cone (dashed gray, $E_{0}^{*} \approx 0.4 \mathrm{eV}$ ) corresponds to overgrown areas covered by MLG. The blue star in (b) and (f) highlights backfolding of the silver band via the graphene reciprocal lattice vectors. Photon energies: (a), (c)-(e) $21.22 \mathrm{eV}$ and (b), (g) $40.81 \mathrm{eV}$.

The huge diffusion mobility of silver on graphene [33,34] leads to immediate clusterization upon room-temperature deposition, yet $\mathrm{Ag}$ intercalation sets in at $\approx 500^{\circ} \mathrm{C}$. The on-top silver clusters start to reevaporate around $600^{\circ} \mathrm{C}$ whereas deintercalation becomes relevant from $\approx 650^{\circ} \mathrm{C}$. By hitting this upper temperature threshold we achieve a satisfactory intercalation scenario while undesired clusters are largely suppressed. ARPES was performed using monochromatized He I (He II) photons with an energy of $h v=21.22 \mathrm{eV}(40.81 \mathrm{eV})$ incident in the detection plane of a 2D hemispherical electron spectrometer (Specs Phoibos 150). The maximum energy resolution was $60 \mathrm{meV}$. X-ray photoelectron spectroscopy (XPS) used nonmonochromatized $\mathrm{Mg} K \alpha$ radiation ( $h v=$ $1253.6 \mathrm{eV}$ ). All measurements were performed in ultrahigh vacuum at room temperature and at a pressure $<5 \times 10^{-10}$ mbar.

\section{RESULTS AND DISCUSSION}

Figure 1(a) displays the low-energy electronic structure of Ag-intercalated ZLG along a closed wedge inside the hexagonal Brillouin zone (BZ) while Fig. 1(b) presents the $\overline{\Gamma K}_{\mathrm{Gr}}$ and $\overline{\Gamma M}_{\mathrm{Gr}}$ directions at a different photon energy. Besides the dominant $\pi$ bands of quasi-free-standing (QF) monolayer graphene (MLG) near $\overline{\mathrm{K}}_{\mathrm{Gr}}$, one additional band can clearly be discerned. It disperses through a maximum $0.59 \pm 0.03 \mathrm{eV}$ below the Fermi level $E_{\mathrm{F}}$ and a saddle point at a binding energy of $E=1.60 \pm 0.03 \mathrm{eV}$ until merging with the $\mathrm{SiC}$ bulk bands around normal emission. Such a feature is absent in pristine ZLG [35], MLG [36], as well as QFMLG decoupled via hydrogen intercalation [19] and must therefore be related to the intercalated silver. Yet, with a valence band maximum (VBM) well below $E_{\mathrm{F}}$, it demonstrates semiconducting behavior as opposed to bulk silver-a prototype $s p$ metal.

The presence of one single Ag-associated band whose dispersion is essentially independent of photon energy [Figs. 1(a) and 1(b)] suggests the large-area ML epitaxy of silver. The positions of the VBM and the saddle point in momentum space indicate a hexagonal $\mathrm{Ag} \mathrm{BZ}$ that is rotated by $30^{\circ}$ with respect to graphene and coincides with the $\mathrm{SiC}$ surface $\mathrm{BZ}$ [Fig. 1(f)]. Therefore, the electronic structure is consistent with a $(1 \times 1)$ epitaxial relationship of silver relative to $\mathrm{SiC}$, i.e., the formation of a triangular lattice ML as with other intercalants [27-29]. The faint intensity marked by the blue star in Fig. 1(b) is due to umklapp scattering of the Ag valence band into the vicinity of $\bar{\Gamma}$ via the reciprocal lattice vectors of graphene- a direct consequence of the long-range order of the combined system.

The isoenergy cuts of Figs. 1(c)-1(e) highlight the topology of the induced band which transforms from a single electron pocket centered at $\bar{\Gamma}$ through the saddle point at $\overline{\mathrm{M}}_{\mathrm{Ag}}$ into two hole pockets peaking into the VBM at $\overline{\mathrm{K}}_{\mathrm{Ag}}$. A similar but metallic surface band exists for silicene grown on $\mathrm{Ag}(111)$ where it derives from $\mathrm{Ag} s p$ electrons hybridizing with Si orbitals [37-40]. Vice versa, our silver ML resides on a hexagonal, Si-terminated surface, therefore suggesting the same microscopic origin of the induced band. Relative to the nearest-neighbor interatomic distance of bulk $\mathrm{Ag}$, the triangular lattice $\mathrm{ML}$ on $\mathrm{SiC}$ is strained by $\approx 6 \%$. While preserving the orbital overlap in terms of band structure formation, together with the influence of the substrate, this leads to obvious deviations from the nearly free $s p$ character of bulk silver [41]. 
The $\mathrm{Ag}$ valence band dispersion $E\left(k_{x}, k_{y}\right)$ can largely be described by a $2 \mathrm{D}$ next-nearest neighbor, $s$-orbital tightbinding (TB) model on the triangular lattice defined by the $\mathrm{SiC}(0001)$ surface (lattice constant $a=3.07 \AA$ [42]),

$$
E\left(k_{x}, k_{y}\right)=E_{0}-\gamma_{1} h_{1}\left(k_{x}, k_{y}\right)-\gamma_{2} h_{2}\left(k_{x}, k_{y}\right),
$$

with

$$
\begin{aligned}
& h_{1}\left(k_{x}, k_{y}\right)=2 \cos \left(k_{x} a\right)+4 \cos \left(k_{x} a / 2\right) \cos \left(\sqrt{3} k_{y} a / 2\right), \\
& h_{2}\left(k_{x}, k_{y}\right)=2 \cos \left(\sqrt{3} k_{y} a\right)+4 \cos \left(\sqrt{3} k_{y} a / 2\right) \cos \left(3 k_{x} a / 2\right) .
\end{aligned}
$$

A fit of Eq. (1) to the spectral maxima of Fig. 1(a) for $\mid k_{\|}-$ $\bar{\Gamma} \mid \geqslant 0.8 \AA^{-1}$ yields a band bottom $E_{0}=5.1 \mathrm{eV}$ as well as nearest- and next-nearest-neighbor hopping amplitudes $\gamma_{1}=$ $0.50 \mathrm{eV}$ and $\gamma_{2}=-0.06 \mathrm{eV}$, respectively. The blue curves represent the fitted TB dispersion throughout Figs. 1(a)-1(e). Away from $\bar{\Gamma}$ the agreement with the experimental data is excellent for both photon energies, confirming the epitaxial alignment of the intercalated silver atoms with respect to $\mathrm{SiC}$ and their true ML character. Around normal emission the model naturally differs as it does not involve the SiC bulk bands and their hybridization with the $\mathrm{Ag}$ states. The latter becomes evident from the disproportionate spectral weight loss of the silver band near $\bar{\Gamma}$ for $h v=40.81 \mathrm{eV}$ [Fig. 1(b)]. The influence of the $\mathrm{SiC}$ substrate is even more extensive in shaping the $\mathrm{Ag}$ dispersion up to the $\mathrm{BZ}$ border, e.g., warping the isoenergy contour at the saddle-point energy [Fig. 1(d)]. This can be explained by electron hopping not being restricted to within the silver ML but also occurring via the substrate. In an effective intralayer TB description such interlayer interactions can lead to negative hopping terms [43] — just as is the case for $\gamma_{2}$ as per Eq. (1).

We note that no $(1 \times 1)-\mathrm{Ag}$ superstructure on hexagonal $\mathrm{SiC}$ has yet been reported [44], while 2D growth does not even occur for cubic SiC [45]. The large-area stability of the triangular lattice ML thus relies on the vertical confinement via the ZLG which transforms into QFMLG upon intercalation [19]. Figure $1(\mathrm{~g})$ displays the resulting Dirac cone centered at $\overline{\mathrm{K}}_{\mathrm{Gr}}$ with a Dirac point binding energy of $E_{0}=0.78 \pm 0.03 \mathrm{eV}$. This corresponds to a moderate $n$-type carrier density of $\approx 4.5 \times 10^{13} \mathrm{~cm}^{-2}$, i.e., a charge transfer of +0.01 electrons per graphene $\mathrm{C}$ atom. Apart from doping, the QFMLG dispersion remains essentially unperturbed by the presence of silver, rendering the combined system a weakly interacting 2D metal/semiconductor van der Waals heterostructure.

The dashed red lines in Fig. 1(g) represent linear extrapolations of the upper (lower) $\pi$ band for $E \leqslant 0.5 \mathrm{eV}(\geqslant 1.2 \mathrm{eV})$. The bands do not cross at the same energy as known from other (intercalated) graphene systems where plasmaron quasiparticle formation was proposed $[36,46,47]$. Resulting from electron-plasmon coupling, the Dirac cone is intertwined with a plasmaron replica and the Dirac point region elongates with increasing coupling strength as detailed in, e.g., Ref. [47]. Since the Ag ML is semiconducting, only partial screening of the electron-plasmon interaction can be expected, consistent with the pronounced elongation $>0.2 \mathrm{eV}$ in Fig. 1(g).

The dashed gray lines in Fig. 1(g) highlight a second, faint cone with a branch crossing at $E_{0}^{*}=0.39 \pm 0.03 \mathrm{eV}$. It stems from regions where ZLG was already covered by an additional graphene layer (i.e., MLG) due to overgrowth at step edges [18]. The doping of these minority regions is consistent with pristine MLG/SiC [36,48], suggesting that they remain nonintercalated.

First-principles calculations of Ag-intercalated ZLG have been performed assuming $(\sqrt{3} \times \sqrt{3}) \mathrm{R} 30^{\circ}-\mathrm{SiC}$ that accommodates $(2 \times 2)$ strained graphene unit cells [49]. In contrast to our findings, they reveal an Ag-related valence band crossing $E_{\mathrm{F}}$. Yet, the doping level of the graphene layer matches the present experiment quite well. Such differences in relative band alignment are plausible since the intercalated atoms seem to interact predominantly with $\mathrm{SiC}$ rather than QFMLG. Hence, it is the nature of the $\mathrm{Ag} / \mathrm{SiC}$ interface that governs the associated dispersion rather than the QFMLG/Ag interface. Our data show no sign of hybridization between the graphene and silver bands, whereas anticrossing gaps of at most $\approx 0.1 \mathrm{eV}$ open in Ref. [49]. While our experimental setup cannot resolve such narrow features unambiguously, they might also be artifacts caused by the hypothetical choice of unit cell.

Au-intercalated ZLG hosts a similar valence band whose maximum and saddle point are identified only 0.05 and $0.4 \mathrm{eV}$ below $E_{\mathrm{F}}$, respectively [29]. Yet, the Dirac point energy $E_{0}$ is almost identical to the present case, owing to the quite similar valence electron configurations of gold and silver. Nevertheless, the precise adsorption geometry of the intercalant and the strain within the triangular ML might well differ between the two systems. This can explain the quantitatively different dispersions, where only in the case of silver the VBM is far away from $E_{\mathrm{F}}$.

Figure 2(a) displays the Ag $3 d$ XPS spectra for $\approx 6 \mathrm{~nm}$ of silver deposited on pristine ZLG without annealing (top) and after intercalation (bottom). The red curves represent fits of a single doublet $\left(m_{j}=5 / 2\right.$ and $\left.3 / 2\right)$ with a spin-orbit splitting fixed at $6.0 \mathrm{eV}$ and an area ratio of $3: 2$. Upon intercalation the $3 d$ doublet shifts by $0.56 \pm 0.10 \mathrm{eV}$ towards higher binding energy due to a different bonding situation resulting from the triangular $\mathrm{Ag}$ lattice and its interaction with the $\mathrm{Si}$ dangling bonds of the substrate. Similar chemical shifts relative to bulk silver occur in $(\sqrt{3} \times \sqrt{3})-\mathrm{Ag} / \mathrm{Si}(111)[50,51]$, qualitatively supporting this interpretation. After intercalation still only a single silver species can be discerned, albeit with slightly increased peak widths. This is another argument for an epitaxial Ag ML, where all atoms are supposed to share the same chemical environment. The shoulder L in the top spectrum of Fig. 2(a) is a characteristic energy loss feature of the $3 d_{5 / 2}$ peak in metallic silver [52]. It is therefore absent in the $3 d$ spectrum of intercalated Ag, underpinning its semiconducting character.

The C $1 s$ spectrum of ZLG is displayed in the top part of Fig. 2(b). The fit considers a SiC bulk component B and two peaks S1, S2 representing the $6 \sqrt{3}$ buffer layer with its partial covalent bonding to $\mathrm{SiC}[35,48]$. In accordance with ARPES, an additional component $\mathrm{O}$ accounts for the overgrown sample areas covered by MLG [53]. From the area ratio of S1, S2 and O we estimate a MLG coverage $\rho_{\mathrm{MLG}}$ of about $10 \%$. Upon intercalation (bottom) the bulk peak B shifts by $0.63 \pm 0.10 \mathrm{eV}$ to lower binding energies and S1, S2 are replaced by a single metallic QFMLG component. This is characteristic of a successful decoupling of the buffer layer via saturation of the Si dangling bonds by the intercalant and 


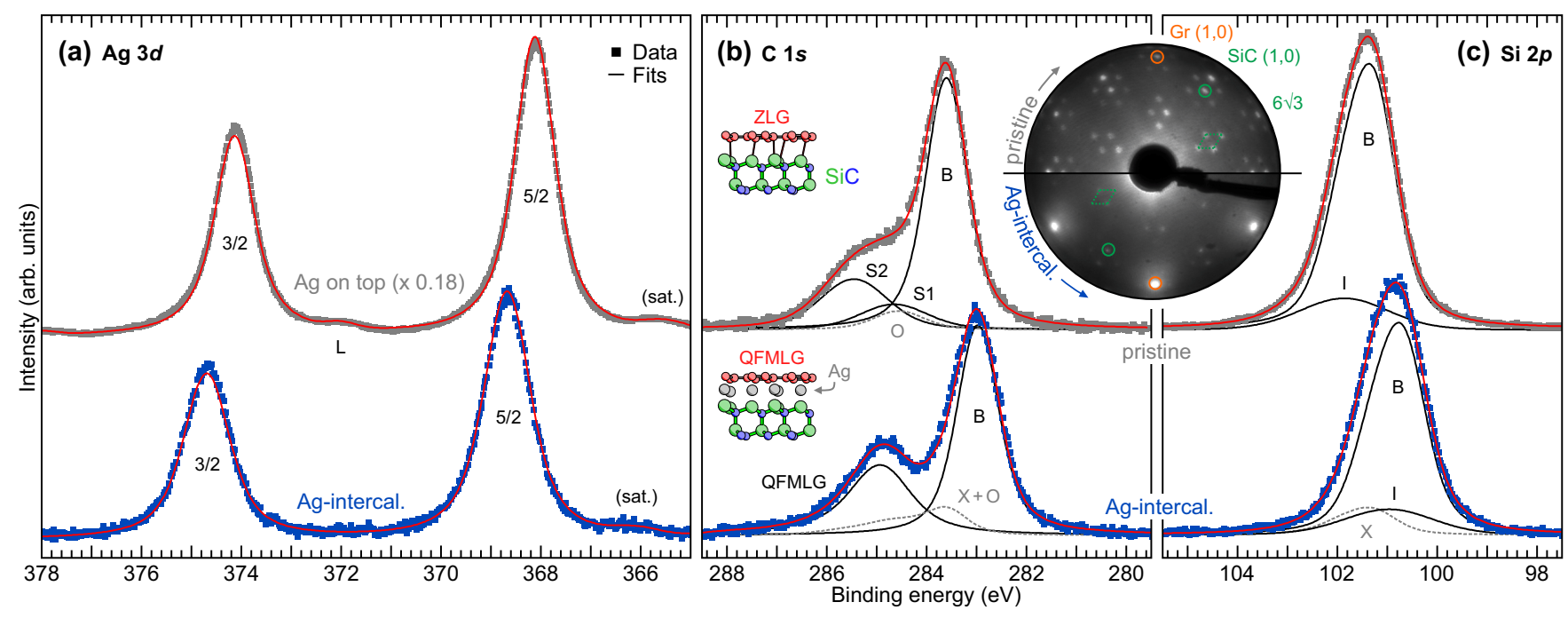

FIG. 2. Fitted XPS spectra of (a) Ag 3d, (b) C $1 s$, and (c) Si $2 p$ for pristine (top) and Ag-intercalated graphene (bottom). The top spectrum in (a) corresponds to $6 \mathrm{~nm}$ of silver deposited on ZLG. The individual fit components are explained in the text. The tiny peak around $366 \mathrm{eV}$ in (a) is a $\mathrm{Mg} \mathrm{K} \alpha_{3}$ satellite of $\mathrm{Ag} 3 d_{3 / 2}$ [52]. The insets in (b) schematically depict the atomic structure of the system. The respective LEED patterns at $67.5 \mathrm{eV}$ are shown in the right inset.

the concomitant formation of quasi-free-standing monolayer graphene [atomic structure schemes in Fig. 2(b) insets]. While the change in band bending at the $\mathrm{SiC}$ surface [54] governs the peak shift of B, the same qualitative behavior applies to ZLG intercalation in general $[19,25,48,55]$. The binding energy of QFMLG exceeds that of peak O (overgrown MLG) by $0.36 \pm$ $0.10 \mathrm{eV}$, matching the energy difference of the Dirac points $\left[E_{0}-E_{0}^{*}=0.39\right.$ eV, Fig. $\left.1(\mathrm{~g})\right]$. Component $\mathrm{X}+\mathrm{O}$ accounts for nonintercalated areas left behind at the sample surface. It represents the sum of peak $\mathrm{O}$ and the scaled residual line shape before intercalation. Scaling of the order of $\rho_{\text {MLG }}$ yields a good fit, consistent with the idea that $\approx 90 \%$ of the sample intercalates while the overgrown regions remain pristine.

The pristine Si $2 p$ spectrum [top part of Fig. 2(c)] is fitted by two doublets $\left(m_{j}=3 / 2\right.$ and $1 / 2$, spin-orbit splitting $0.62 \mathrm{eV}$, area ratio $2: 1$ ), one each for bulk (B) and interfacial $\mathrm{Si}$ (I). The binding energy of I is $0.42 \pm 0.10 \mathrm{eV}$ higher caused by covalent bonding to ZLG [48]. After intercalation (bottom) peak B has shifted by $0.61 \pm 0.10 \mathrm{eV}$ towards lower binding energies, in agreement with the characteristic evolution of the $\mathrm{C} 1 s$ spectrum in terms of surface band bending. Component I shifts by $0.90 \pm 0.10 \mathrm{eV}$ in the same direction, pointing towards a covalent interaction between the interfacial $\mathrm{Si}$ and the intercalated silver atoms just as ARPES and the $\mathrm{Ag} 3 d$ core levels. The decrease in binding energy of I relative to B upon the decoupling of ZLG seems plausible considering the higher electronegativity of $\mathrm{C}$ vs $\mathrm{Ag}$ [56]. Component $X$ again represents the downscaled pristine line shape and its fitted residual share of 0.12 turns out consistent with the large-area intercalation scenario.

The latter is also reflected in the evolution of the lowenergy electron diffraction (LEED) pattern (right inset of Fig. 2). For pristine ZLG the first-order beams of graphene and $\mathrm{SiC}$ are accompanied by multiple superstructure spots, all of which are associated with the $(6 \sqrt{3} \times 6 \sqrt{3}) \mathrm{R} 30^{\circ}$ buffer layer reconstruction [48]. Upon intercalation the latter is largely lifted such that the $\mathrm{SiC}$ and $6 \sqrt{3}$ spots are strongly suppressed while intense graphene $(1,0)$ spots emerge, oversaturating the grayscale $[19,25,48,55]$. The nonintercalated areas $(\approx 10 \%)$ definitely add to the faint residual $6 \sqrt{3}$ pattern. However, also intercalated regions contribute as the full periodicity of the system is retained and multiple scattering at the graphene and $\mathrm{SiC}$ planes produces the same superstructure [57]. Just as expected from the $(1 \times 1)$ alignment of $\mathrm{Ag}$ relative to $\mathrm{SiC}$, additional diffraction spots of different periodicity cannot be discerned.

Based on the data of Figs. 1 and 2 we finally discuss the sample homogeneity. Large areas $(\approx 90 \%$, see above) are intercalated, hosting the Dirac cone of QFMLG [Fig. 1(g)]. If the observed valence band [Figs. 1(a)-1(e)] were not induced by intercalated silver below QFMLG, it could only be explained by an Ag monolayer adsorbed on top. This scenario can readily be excluded as it neither promotes the orbital overlap necessary for the apparent hybridization with the $\mathrm{SiC}$ bands nor does it facilitate a $(1 \times 1)$ alignment relative to $\mathrm{SiC}$. A distinct Ag $3 d$ component would further appear due to the different chemical environment compared to intercalated silver. We thus conclude that the QFMLG and Ag bands originate from the same $\approx 90 \%$ of the sample surface and represent a true metal/semiconductor heterostructure.

\section{CONCLUSION}

In summary, we have demonstrated the band structure formation of an epitaxial triangular lattice monolayer of silver realized via intercalation at the interface of $\mathrm{SiC}$ and its $(6 \sqrt{3} \times 6 \sqrt{3}) \mathrm{R} 30^{\circ}$ carbon buffer layer. A simple 2D tightbinding model quantitatively reproduces the $\mathrm{Ag}$ valence band dispersion close to the Brillouin zone border with a saddle point at $\overline{\mathrm{M}}_{\mathrm{Ag}}$ and the valence band maximum at $\overline{\mathrm{K}}_{\mathrm{Ag}}$. The latter resides $0.59 \mathrm{eV}$ below the Fermi level, rendering the 2D silver monolayer semiconducting as opposed to the bulk metal. We observe strong hybridization with $\mathrm{SiC}$ bands near $\bar{\Gamma}$, reflecting the substantial interaction of the intercalant with 
its substrate as corroborated by core level spectroscopy. The intercalated $\mathrm{Ag}$ monolayer is readily combined into a $2 \mathrm{D}$ heterostructure with $n$-doped quasi-free-standing graphene. Our study helps to establish epitaxial graphene on $\mathrm{SiC}$ as a convenient platform for the scalable synthesis of monoelemental monolayers which hold great promise for a wide range of next-generation applications.

\section{ACKNOWLEDGMENTS}

The authors would like to thank Dirk Manske for fruitful discussions about the tight-binding model. This work was supported by the Deutsche Forschungsgemeinschaft (DFG) through Sta315/9-1.
[1] S. Z. Butler, S. M. Hollen, L. Cao, Y. Cui, J. A. Gupta, H. R. Gutiérrez, T. F. Heinz, S. S. Hong, J. Huang, A. F. Ismach, E. Johnston-Halperin, M. Kuno, V. V. Plashnitsa, R. D. Robinson, R. S. Ruoff, S. Salahuddin, J. Shan, L. Shi, M. G. Spencer, M. Terrones, W. Windl, and J. E. Goldberger, ACS Nano 7, 2898 (2013).

[2] K. S. Novoselov, A. Mishchenko, A. Carvalho, and A. H. C. Neto, Science 353, aac9439 (2016).

[3] F. Koppens, T. Mueller, P. Avouris, A. Ferrari, M. Vitiello, and M. Polini, Nat. Nanotechnol. 9, 780 (2014).

[4] G. Fiori, F. Bonaccorso, G. Iannaccone, T. Palacios, D. Neumaier, A. Seabaugh, S. K. Banerjee, and L. Colombo, Nat. Nanotechnol. 9, 768 (2014).

[5] D. Deng, K. Novoselov, Q. Fu, N. Zheng, Z. Tian, and X. Bao, Nat. Nanotechnol. 11, 218 (2016).

[6] N. Mounet, M. Gibertini, P. Schwaller, D. Campi, A. Merkys, A. Marrazzo, T. Sohier, I. E. Castelli, A. Cepellotti, G. Pizzi, and N. Marzari, Nat. Nanotechnol. 13, 246 (2018).

[7] B. Lalmi, H. Oughaddou, H. Enriquez, A. Kara, S. Vizzini, B. Ealet, and B. Aufray, Appl. Phys. Lett. 97, 223109 (2010).

[8] M. Dávila, L. Xian, S. Cahangirov, A. Rubio, and G. Le Lay, New J. Phys. 16, 095002 (2014).

[9] F.-f. Zhu, W.-j. Chen, Y. Xu, C.-1. Gao, D.-d. Guan, C.-h. Liu, D. Qian, S.-C. Zhang, and J.-f. Jia, Nat. Mater. 14, 1020 (2015).

[10] J. Ji, X. Song, J. Liu, Z. Yan, C. Huo, S. Zhang, M. Su, L. Liao, W. Wang, Z. Ni, Y. Hao, and H. Zeng, Nat. Commun. 7, 13352 (2016).

[11] F. Reis, G. Li, L. Dudy, M. Bauernfeind, S. Glass, W. Hanke, R. Thomale, J. Schäfer, and R. Claessen, Science 357, 287 (2017).

[12] J. Shah, W. Wang, H. M. Sohail, and R. I. G. Uhrberg, 2D Mater. 7, 025013 (2020).

[13] Y. Chen, Z. Fan, Z. Zhang, W. Niu, C. Li, N. Yang, B. Chen, and H. Zhang, Chem. Rev. 118, 6409 (2018).

[14] T. Ling, J.-J. Wang, H. Zhang, S.-T. Song, Y.-Z. Zhou, J. Zhao, and X.-W. Du, Adv. Mater. 27, 5396 (2015).

[15] B. An, M. Li, J. Wang, and C. Li, Front. Chem. Sci. Eng. 10, 360 (2016).

[16] I. Forbeaux, J. M. Themlin, and J. M. Debever, Phys. Rev. B 58, 16396 (1998).

[17] C. Berger, Z. Song, T. Li, X. Li, A. Y. Ogbazghi, R. Feng, Z. Dai, A. N. Marchenkov, E. H. Conrad, P. N. First, and W. A. de Heer, J. Phys. Chem. B 108, 19912 (2004).

[18] K. V. Emtsev, A. Bostwick, K. Horn, J. Jobst, G. L. Kellogg, L. Ley, J. L. McChesney, T. Ohta, S. A. Reshanov, J. Röhrl, E. Rotenberg, A. K. Schmid, D. Waldmann, H. B. Weber, and Th. Seyller, Nat. Mater. 8, 203 (2009).

[19] C. Riedl, C. Coletti, T. Iwasaki, A. A. Zakharov, and U. Starke, Phys. Rev. Lett. 103, 246804 (2009).
[20] I. Gierz, T. Suzuki, R. T. Weitz, D. S. Lee, B. Krauss, C. Riedl, U. Starke, H. Höchst, J. H. Smet, C. R. Ast, and K. Kern, Phys. Rev. B 81, 235408 (2010).

[21] K. V. Emtsev, A. A. Zakharov, C. Coletti, S. Forti, and U. Starke, Phys. Rev. B 84, 125423 (2011).

[22] A. L. Walter, K.-J. Jeon, A. Bostwick, F. Speck, M. Ostler, Th. Seyller, L. Moreschini, Y. S. Kim, Y. J. Chang, K. Horn, and E. Rotenberg, Appl. Phys. Lett. 98, 184102 (2011).

[23] S. Forti, A. Stöhr, A. A. Zakharov, C. Coletti, K. V. Emtsev, and U. Starke, 2D Mater. 3, 035003 (2016).

[24] J. L. McChesney, A. Bostwick, T. Ohta, Th. Seyller, K. Horn, J. González, and E. Rotenberg, Phys. Rev. Lett. 104, 136803 (2010).

[25] Ph. Rosenzweig, H. Karakachian, S. Link, K. Küster, and U. Starke, Phys. Rev. B 100, 035445 (2019).

[26] S. Link, S. Forti, A. Stöhr, K. Küster, M. Rösner, D. Hirschmeier, C. Chen, J. Avila, M. C. Asensio, A. A. Zakharov, T. O. Wehling, A. I. Lichtenstein, M. I. Katsnelson, and U. Starke, Phys. Rev. B 100, 121407(R) (2019).

[27] S. Hayashi, A. Visikovskiy, T. Kajiwara, T. Iimori, T. Shirasawa, K. Nakastuji, T. Miyamachi, S. Nakashima, K. Yaji, K. Mase, F. Komori, and S. Tanaka, Appl. Phys. Express 11, 015202 (2017).

[28] K. Yaji, A. Visikovskiy, T. Iimori, K. Kuroda, S. Hayashi, T. Kajiwara, S. Tanaka, F. Komori, and S. Shin, Phys. Rev. Lett. 122, 126403 (2019).

[29] S. Forti, S. Link, A. Stöhr, Y. Niu, A. A. Zakharov, C. Coletti, and U. Starke, Nat Commun 11, 2236 (2020).

[30] N. Briggs, Z. M. Gebeyehu, A. Vera, T. Zhao, K. Wang, A. De La Fuente Duran, B. Bersch, T. Bowen, K. L. Knappenberger, and J. A. Robinson, Nanoscale 11, 15440 (2019).

[31] S. Soubatch, S. E. Saddow, S. P. Rao, W. Lee, M. Konuma, and U. Starke, Mater. Sci. Forum 483, 761 (2005).

[32] Monitored by an infrared pyrometer (emissivity of 0.63 ).

[33] X. Liu, C.-Z. Wang, M. Hupalo, W. C. Lu, M. C. Tringides, Y. X. Yao, and K. M. Ho, Phys. Chem. Chem. Phys. 14, 9157 (2012).

[34] X. Liu, C.-Z. Wang, M. Hupalo, H.-Q. Lin, K.-M. Ho, and M. Tringides, Crystals 3, 79 (2013).

[35] K. V. Emtsev, F. Speck, Th. Seyller, L. Ley, and J. D. Riley, Phys. Rev. B 77, 155303 (2008).

[36] A. Bostwick, T. Ohta, Th. Seyller, K. Horn, and E. Rotenberg, Nat. Phys. 3, 36 (2007).

[37] D. Tsoutsou, E. Xenogiannopoulou, E. Golias, P. Tsipas, and A. Dimoulas, Appl. Phys. Lett. 103, 231604 (2013).

[38] S. K. Mahatha, P. Moras, V. Bellini, P. M. Sheverdyaeva, C. Struzzi, L. Petaccia, and C. Carbone, Phys. Rev. B 89, 201416(R) (2014). 
[39] X. Xu, J. Zhuang, Y. Du, H. Feng, N. Zhang, C. Liu, T. Lei, J. Wang, M. Spencer, T. Morishita, X. Wang, and S. X. Dou, Sci. Rep. 4, 7543 (2014).

[40] P. M. Sheverdyaeva, S. K. Mahatha, P. Moras, L. Petaccia, G. Fratesi, G. Onida, and C. Carbone, ACS Nano 11, 975 (2016).

[41] L.-M. Yang, T. Frauenheim, and E. Ganz, Phys. Chem. Chem. Phys. 17, 19695 (2015).

[42] A. Taylor and R. M. Jones, in Silicon Carbide-A High Temperature Semiconductor, edited by J. R. O'Connor and J. Smiltens (Pergamon, Oxford, UK, 1960), p. 147.

[43] E. Pavarini, I. Dasgupta, T. Saha-Dasgupta, O. Jepsen, and O. K. Andersen, Phys. Rev. Lett. 87, 047003 (2001).

[44] S. Soubatch and U. Starke, Mater. Sci. Forum 457-460, 419 (2004).

[45] D. W. Niles, H. Höchst, G. W. Zajac, T. H. Fleisch, B. C. Johnson, and J. M. Meese, J. Vac. Sci. Technol. A 6, 1584 (1988).

[46] A. Bostwick, F. Speck, Th. Seyller, K. Horn, M. Polini, R. Asgari, A. H. MacDonald, and E. Rotenberg, Science 328, 999 (2010).

[47] A. L. Walter, A. Bostwick, K.-J. Jeon, F. Speck, M. Ostler, Th. Seyller, L. Moreschini, Y. J. Chang, M. Polini, R. Asgari, A. H. MacDonald, K. Horn, and E. Rotenberg, Phys. Rev. B 84, 085410 (2011).

[48] C. Riedl, C. Coletti, and U. Starke, J. Phys. D: Appl. Phys. 43, 374009 (2010).
[49] C.-H. Hsu, W.-H. Lin, V. Ozolins, and F.-C. Chuang, Appl. Phys. Lett. 100, 063115 (2012).

[50] S. Günther, A. Kolmakov, J. Kovac, L. Casalis, L. Gregoratti, M. Marsi, and M. Kiskinova, Surf. Sci. 377-379, 145 (1997).

[51] M. Hashimoto, F.-Z. Guo, M. Suzuki, M. Ueda, Y. Matsuoka, T. Kinoshita, K. Kobayashi, S. Shin, M. Oura, T. Takeuchi, Y. Saito, T. Matsushita, T. Yasue, and T. Koshikawa, Surf. Interface Anal. 40, 1772 (2008).

[52] J. F. Moulder, W. F. Stickle, P. E. Sobol, and K. D. Bomben, in Handbook of X-ray Photoelectron Spectroscopy: A Reference Book of Standard Spectra for Identification and Interpretation of XPS Data, edited by J. Chastain (Perkin-Elmer Corporation, Physical Electronics Division, Eden Prairie, MN, 1992), p. 120.

[53] For a stable fit the energy shifts of $S 1, S 2$ relative to B and their area ratio of $1: 2$ were fixed according to Ref. [35] .

[54] J. Ristein, S. Mammadov, and Th. Seyller, Phys. Rev. Lett. 108, 246104 (2012).

[55] S. Wolff, S. Roscher, F. Timmermann, M. V. Daniel, F. Speck, M. Wanke, M. Albrecht, and Th. Seyller, Ann. Phys. (Berlin) 531, 1900199 (2019).

[56] CRC Handbook of Chemistry and Physics, edited by W. M. Haynes, D. R. Lide, and T. J. Bruno, 96th ed. (CRC Press, Boca Raton, FL, 2015).

[57] S. Forti, K. V. Emtsev, C. Coletti, A. A. Zakharov, C. Riedl, and U. Starke, Phys. Rev. B 84, 125449 (2011). 